\title{
MICROSTRUCTURED OPTICAL FIBER FOR X-RAY DETECTION
}

\author{
S. L. DeHaven \\ NASA Langley Research Center, Hampton, VA 23681
}

\begin{abstract}
A novel scintillating optical fiber is presented using a composite micro-structured quartz optical fiber. Scintillating materials are introduced into the multiple inclusions of the fiber. This creates a composite optical fiber having quartz as a cladding with an organic scintillating material core. X-ray detection using these fibers is compared to a collimated cadmium telluride (CdTe) detector over an energy range from 10 to $40 \mathrm{keV}$. Results show a good correlation between the fiber count rate trend and that of the CdTe detector.
\end{abstract}

Keywords: X-ray Detector, Fiber Optic, Scintillator

PACS: 07.85.Fv, 29.40.Mc, 42.81.Wg, 42.81.Pa

\section{INTRODUCTION}

Detecting $\mathrm{x}$-rays began with the discovery of Bremstralung radiation and has steadily improved with advances in technology. Many types of x-ray detectors exist including films, ionization chambers, Geiger-Mueller counters, scintillation materials, and semiconductors. Two categories of $\mathrm{x}$-ray detectors addressed in this paper are scintillation and solid state.

Many types of scintillator and solid state materials exist. Scintillation detectors use a liquid or crystal which interacts with $\mathrm{x}$-rays to produce light measured with a photon detector. Solid state detectors use $\mathrm{x}$-ray energy to induce a change in current when $\mathrm{x}$-rays interact with the material.

Scintillator materials can be inorganic or organic. Anthracene is the standard organic crystal and all liquid scintillators are organic. However, they are somewhat fragile, have low efficiency, and require specific environments. Solid state detectors, such as $\mathrm{CdTe}$, have several benefits but require cooling because of dark noise considerations and need an evacuated space behind a beryllium window [1].

The conventional optical fiber for $\mathrm{x}$-ray and particle detection is plastic PMMA scintillating fiber. The plastic fiber uses an organic hydrocarbon scintillator mixed with a polymer core. Because of the relatively high visible wavelength absorption losses in the plastic, the fiber core must be 1000 microns in diameter [2].

The novel approach proposed in this paper is combining a scintillator with microstructured optical fiber to form an $\mathrm{x}$-ray detector. The microstructured optical fiber presented uses solid crystalline anthracene or a liquid hydrocarbon (1,2,4-trimethyl benzene) scintillator inside a quartz matrix. The light guiding mechanism is through total 
internal reflection which is the same for plastic fiber. The visible wavelength absorption for the liquid hydrocarbon and crystalline anthracene is less than plastic scintillating fiber.

A solid state CdTe $\mathrm{x}$-ray detector is used to characterize the $\mathrm{x}$-ray tube source and compare with the fiber output. The geometry of the microstructured optical fiber is considerably smaller than plastic fiber. In the fiber presented, each inclusion is 2.5 microns with 168 inclusions inside a 125 micron outside diameter as shown in Figure 1. This allows standard FC fiber optic connectors to standard multimode optical fiber and detectors.

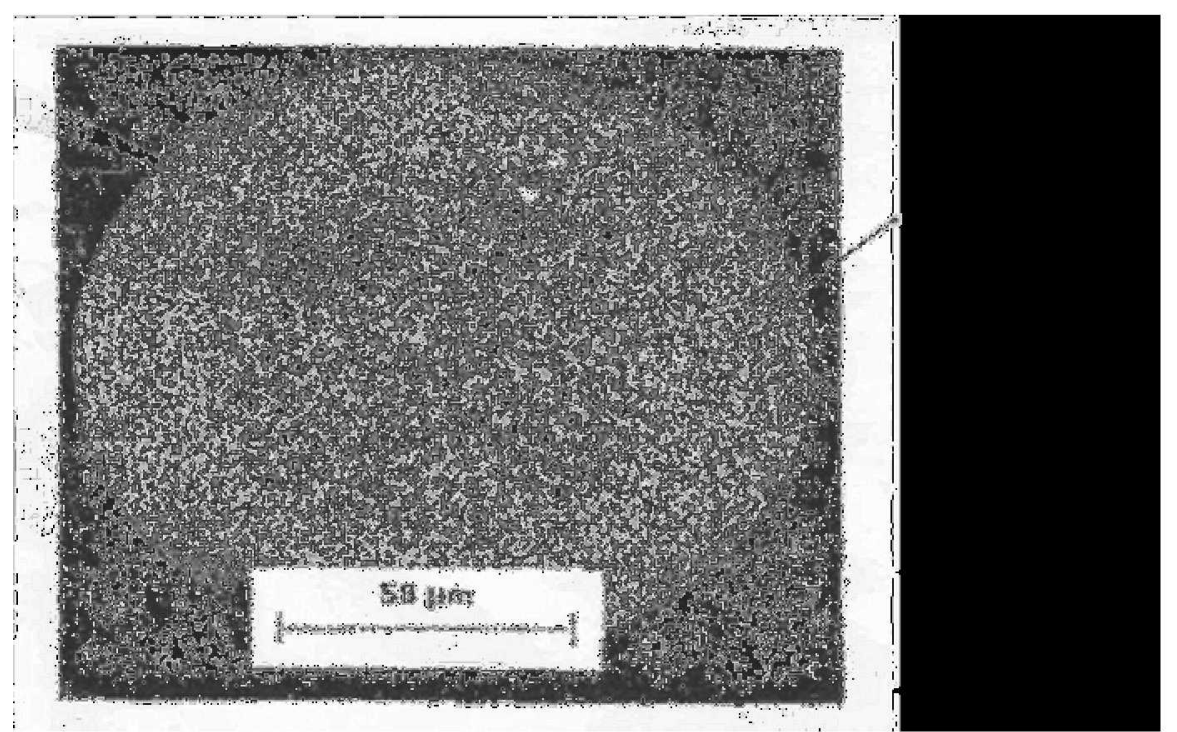

FIGURE 1. Microstructured quartz optical fiber cross-section. Total fiber diameter is 125 microns with 168 air filled inclusions. These inclusions become fiber cores when filled with scintillator material.

\section{THEORY}

The x-ray detecting fiber microstructure is composed of 168 micro-core multimode waveguides, each transmitting $420 \mathrm{~nm}$ wavelength (indigo) visible light. The light is from the energy conversion of short wavelength higher energy $x$-ray photons to a larger quantity of lower energy longer wavelength photons. This fluorescence effect generates spontaneous emission $420 \mathrm{~nm}$ wavelength photons.

The fluorescence from an organic scintillator comes from a two-level $\pi$ orbital $x-$ ray absorption and re-emission of visible light as shown in Figure 2 [1]. This same fluorescence occurs from using ultraviolet (UV) photons instead of x-rays. Figure 3 shows the liquid scintillator UV photon absorption in the $370-390 \mathrm{~nm}$ wavelength range, with reemission at $420 \mathrm{~nm}$.

The scintillator material is placed in the fiber inclusions and guides light using total internal reflection. The scintillator acts as the core with the quartz as cladding. As such, photons travel through individual 2.5 micron diameter cores and each core behaves as a separate waveguide.

The liquid filled fiber operates using a weak guidance waveguide theory. The anthracene crystal fiber can use the same theory but it is less accurate due to anthracene's slightly higher index of refraction. The index of refraction difference between the scintillator and quartz provides the waveguide effect [3].

$\mathrm{X}$-rays and UV light act to provide a pump effect seen in lasers; except in this case there is no population inversion because of the two level absorption and re-emission. As such, this is not a fiber laser but the excitation process to generate light is similar. Photons 
generated due to spontaneous emission fluorescence from $\mathrm{x}$-ray absorption are represented as non-coherent plane waves.

The generation of photons is de-coupled from the waveguide properties. Even though photons are generated in fiber materials, transmission behaves as though from an external source because the emission occurs on a sub-atomic level. This same approach applies to fiber optic lasers, erbium doped fibers, Raman scattering, and Rayleigh scattering effects in optical fiber $[5,6]$.

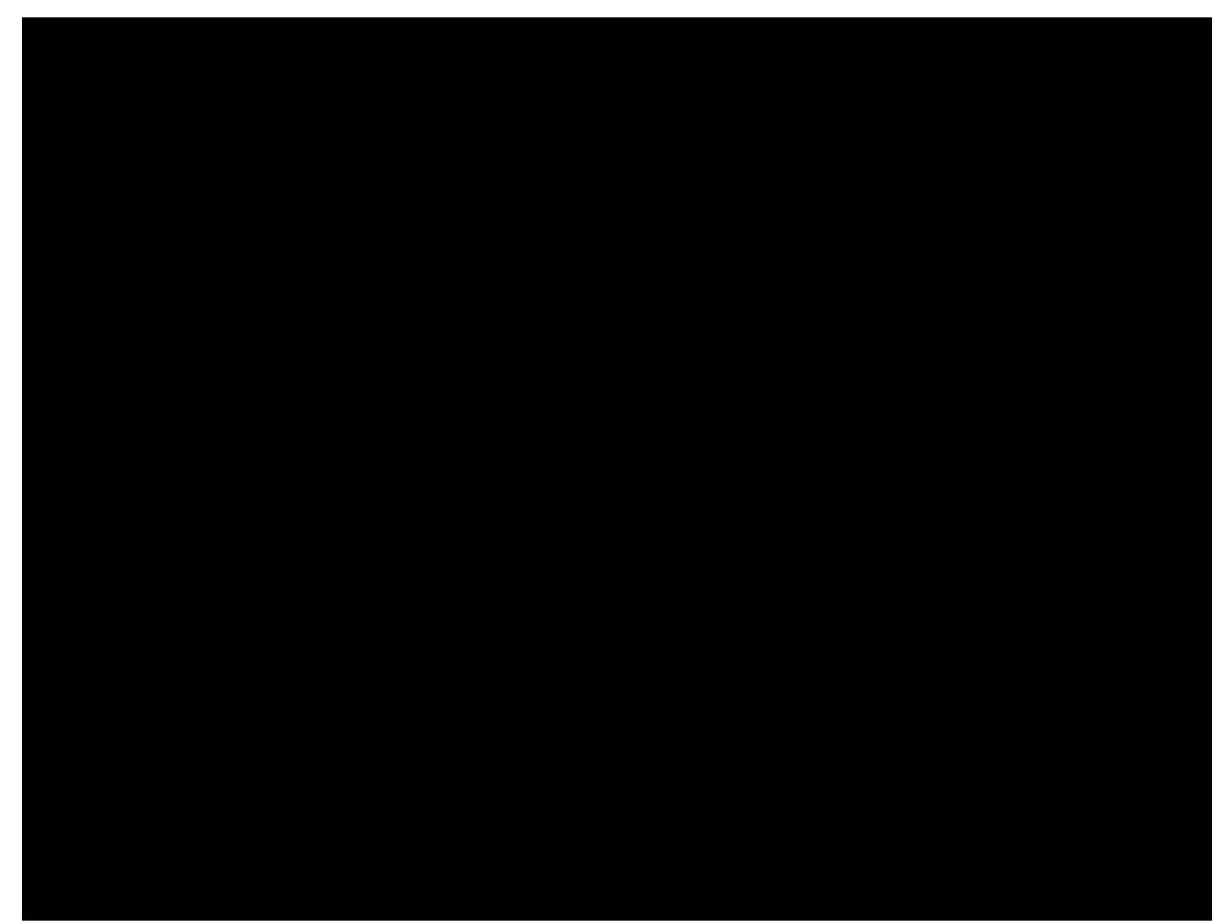

FIGURE 2. Shown is a typical organic scintillator two-level $\pi$ orbital photon energy absorption-reemission diagram.

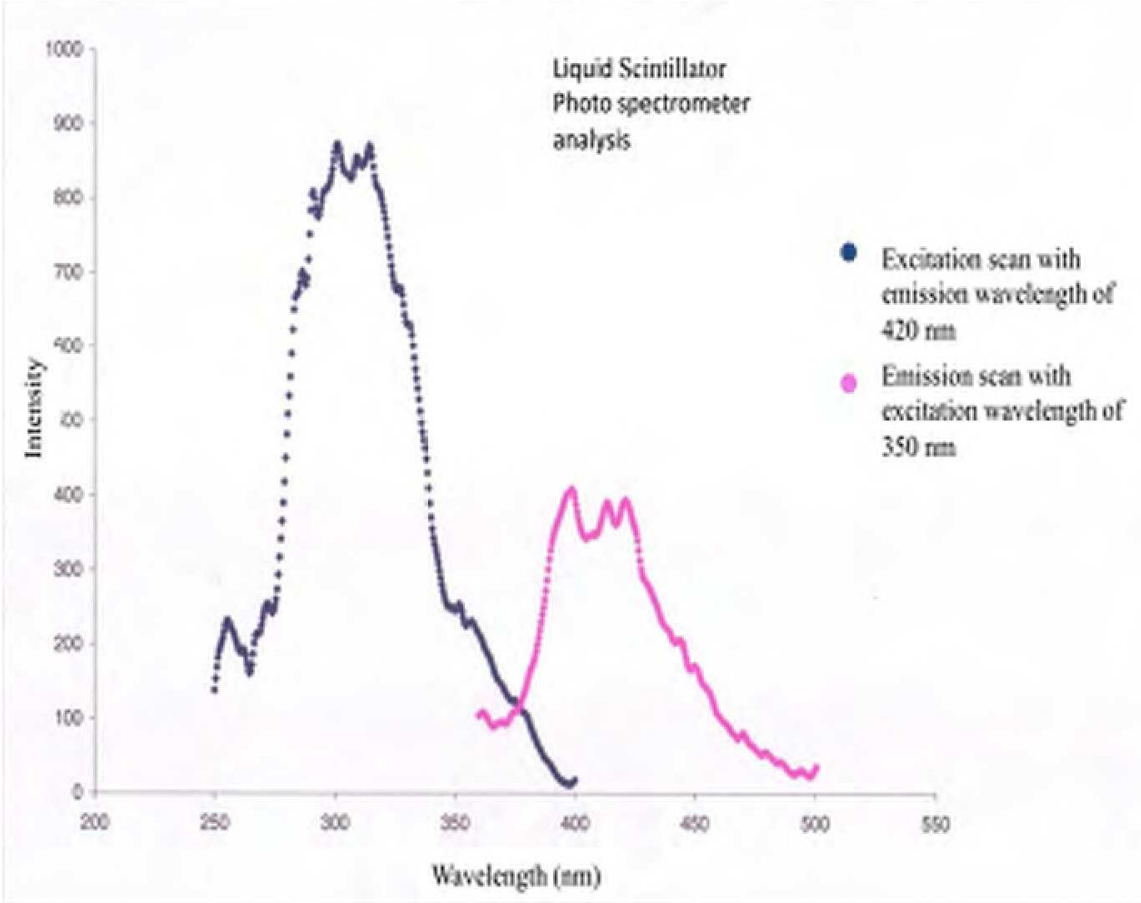

FIGURE 3. Shown are the photospectrometer analysis results. UV light is absorbed and $420 \mathrm{~nm}$ photons are emitted from the liquid scintillator. 
Absorption of the x-rays is based on Beer's law along the axial fiber length using Vinyl toluene property coefficients [4]. The small core and large length to diameter ratio fiber create a geometry where absorption is along the fiber length and provides a directional absorption property.

\section{EXPERIMENT}

Sample preparation for the liquid scintillator fiber was done by putting liquid scintillator into the microstructured optical fiber. This process used capillary action and a pressure gradient by pressurizing the liquid at one end of the fiber. Anthracene crystal fiber was prepared using a modified Bridgman-Stockbarger technique [7]. An approximately $12 \mathrm{~cm}$ long fiber was chosen because that length has over $95 \% \mathrm{x}$-ray photon absorption in the scintillator materials.

Figure 4 shows light generation from a UV lamp source during examination and testing of prepared fibers before exposure to x-rays. As described in theory, light is emitted through the scintillator material with the fiber acting as a bundle of 2.5 micron core sub-fibers. This inspection allows determining expected output from the fiber before exposure to $\mathrm{x}$-rays.

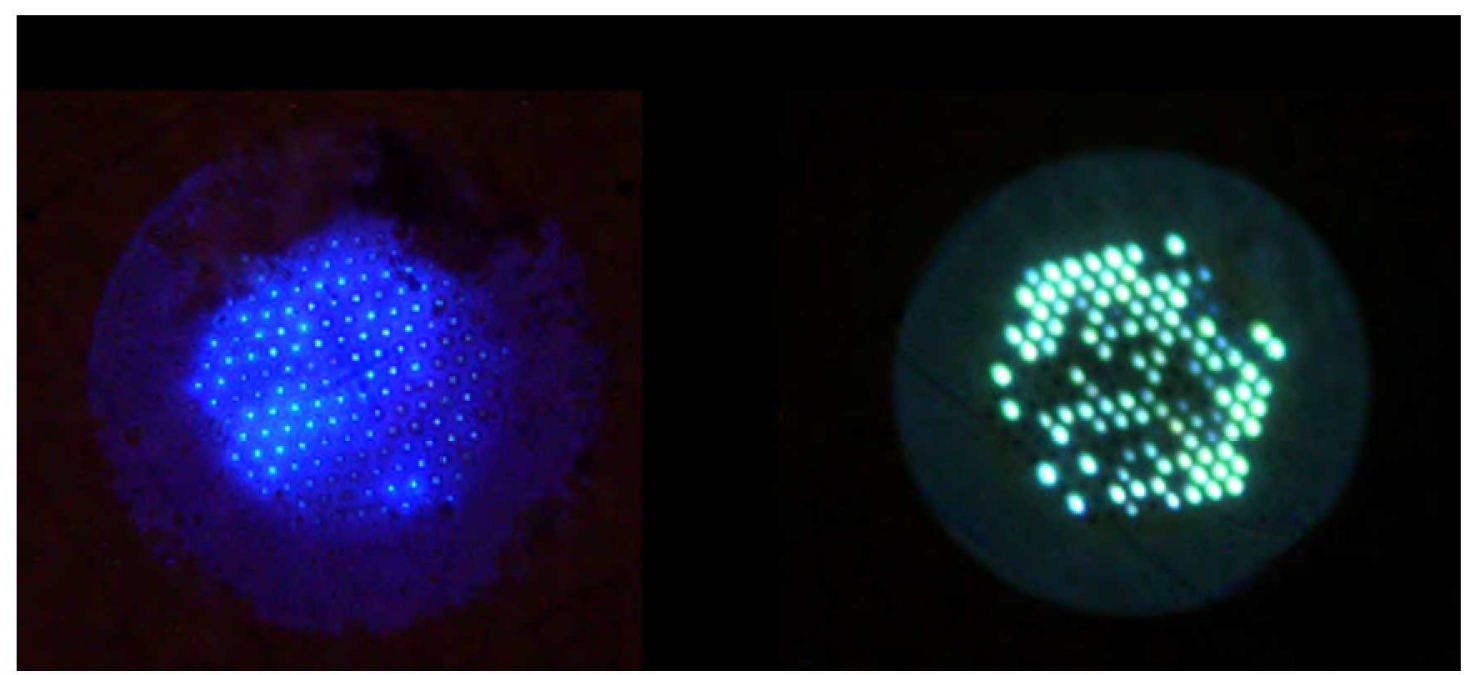

FIGURE 4. Emission of $420 \mathrm{~nm}$ photons using UV excitation on opposite end of fiber, discontinuities in the scintillator material result in no emission from the fiber end.

An experiment was constructed to measure the relative photon counts from the microstructured fibers with scintillating materials. The x-ray tube source was characterized using a CdTe detector and multichannel analyzer. Measurements were taken between $x$-ray tube voltages of 10 and $40 \mathrm{kV}$. A $40 \mathrm{kV}, 4 \mathrm{~W}$ silver anode $\mathrm{x}$-ray tube was used as the source.

Figure 5 shows the experimental setup for $\mathrm{x}$-ray tube characterization and scintillating fiber output measurement. For characterization, the CdTe detector assembly with a collimator collects $\mathrm{x}$-ray photons. During fiber measurement, a photomultiplier tube collects light pulses from the optical fiber. 


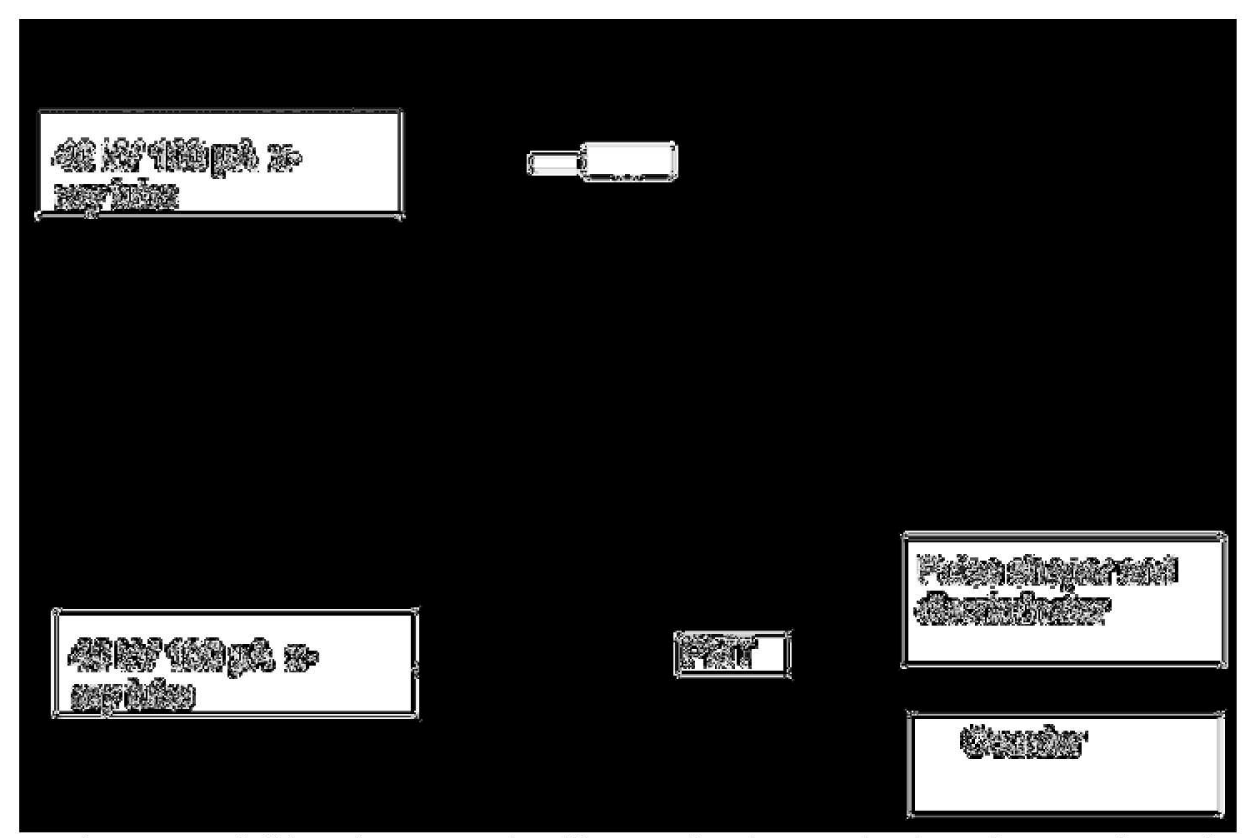

FIGURE 5. Placement of CdTe detector and collimator for characterization of $\mathrm{x}$-ray tube and taking fiber data

The CdTe detector operates with a multi-channel analyzer using a collimator having a $5 \mathrm{~mm}$ thick tungsten disk with a 100 micron diameter aperture followed by a 200 micron diameter aperture disk. The CdTe detector is $100 \%$ efficient at detecting $\mathrm{x}$-ray photons between the energies of $10-50 \mathrm{keV}$. The CdTe detector operates in conjunction with a computer to store and process data.

This $\mathrm{x}$-ray tube characterization was done prior to taking the fiber $\mathrm{x}$-ray scintillation data to compare with the CdTe detector counts. The tube characterization was done at five minute intervals over a series of weeks to ensure tube stability and measurement repeatability. The CdTe detector energy was calibrated done using an Am241 radioactive source. The collimator and tube were carefully aligned to maximize photon counts through the collimator placed $0.5 \mathrm{~cm}$ from the tube.

The prepared fiber was placed in the same location as the detector collimator while varying $x$-ray tube voltage without changing source current during measurements. Scintillating fiber was coupled to the photomultiplier tube FC fiber optic adapter with a bare fiber adapter. The photomultiplier tube module used a bialkali cathode with peak quantum efficiency at $420 \mathrm{~nm}$. A FC fiber optic coupler was supplied with the module. Scintillation inside the fiber was directed to the photomultiplier tube.

Signals from a single photomultiplier tube module went to a discriminator circuit, which discards voltage pulses below an adjustable level. The remaining pulses were sent to a digital pulse counter where the total is accumulated over a five minute time period. This total pulse count was recorded for discrete $\mathrm{x}$-ray tube voltages between 10 and $40 \mathrm{kV}$.

A blackout cloth was used over a leaded Plexiglas three-sided enclosure with a lead-foil covered aluminum back plate. Power and cables to the photomultiplier tube and $x$-ray tube were routed between the Plexiglas enclosure and backing plate. The photomultiplier tube module and the fiber couplers were wrapped in lead to reduce noise.

The blackout cloth reduced stray photons and their subsequent noise but allowed easy access to the experimental setup. Additionally, the room was partially darkened during operation to remove fluorescent light fixture magnetic and photonic noise. Power for the electronics had a common ground. 


\section{RESULTS AND DISCUSSION}

Figure 6 shows the $\mathrm{x}$-ray tube photon energy spectrum collected using the CdTe semiconductor detector with 100 micron collimator to characterize the tube. Tube characterization and fiber data were taken over five minute intervals. A 30 micron thick sheet of aluminum foil was placed in front of the tube as a low energy filter. The collimator and fibers were positioned $0.5 \mathrm{~cm}$ from the tube end in the same relative position.

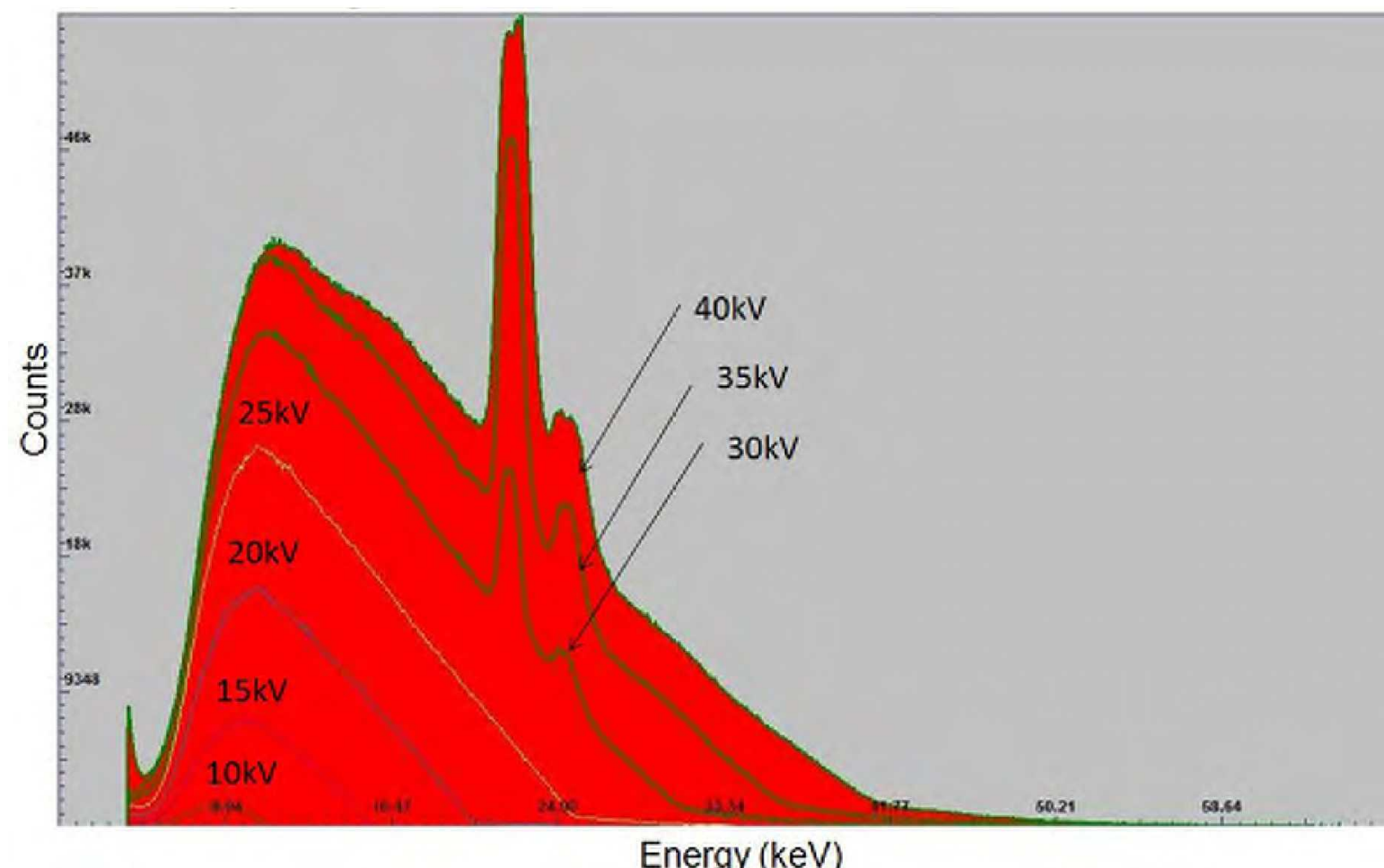

FIGURE 6. $40 \mathrm{kV}$ silver anode $\mathrm{x}$-ray tube characterization with photon number and energy distribution curves at various tube voltages. The plot is of photon counts versus energy (keV) collected using a 100 micron collimator with the CdTe detector over a 300 second time interval.

Figure 7 shows the ratio of liquid and solid filled fiber to CdTe counts vs. tube voltage as a percent efficiency. This percent efficiency trend for both liquid and solid filled fibers increases with the tube voltage. The total number of photons counted from the fiber follows the same trend as the CdTe detector. Anthracene fiber output is higher than the liquid for tube voltages above $30 \mathrm{kV}$ with a $37 \%$ increase at $40 \mathrm{kV}$. The liquid output is rated at $60 \%$ of anthracene.

Some considerations noted in the data are that the total area of the microstructured fiber exposed to x-rays is $10.5 \%$ of the CdTe collimator. Considering the area used, the anthracene fiber efficiency increases to $8.5 \%$ and the liquid to $6.7 \%$ at $40 \mathrm{kV}$. Anthracene has a bulk efficiency of $\sim 5 \%$ and organic scintillator efficiencies operate between $1-5 \%$ [8]. This indicates there is increased efficiency associated with having scintillator material in a microstructured geometry.

This paper shows a novel microstructured optical fiber containing scintillator material can be used to detect $\mathrm{x}$-rays. The fiber quartz protects the scintillator material from the environment and small diameter fiber crystals tolerate bending inside the fiber. While the assembled fiber efficiency is low, the signal to noise ratio is over 25:1. 


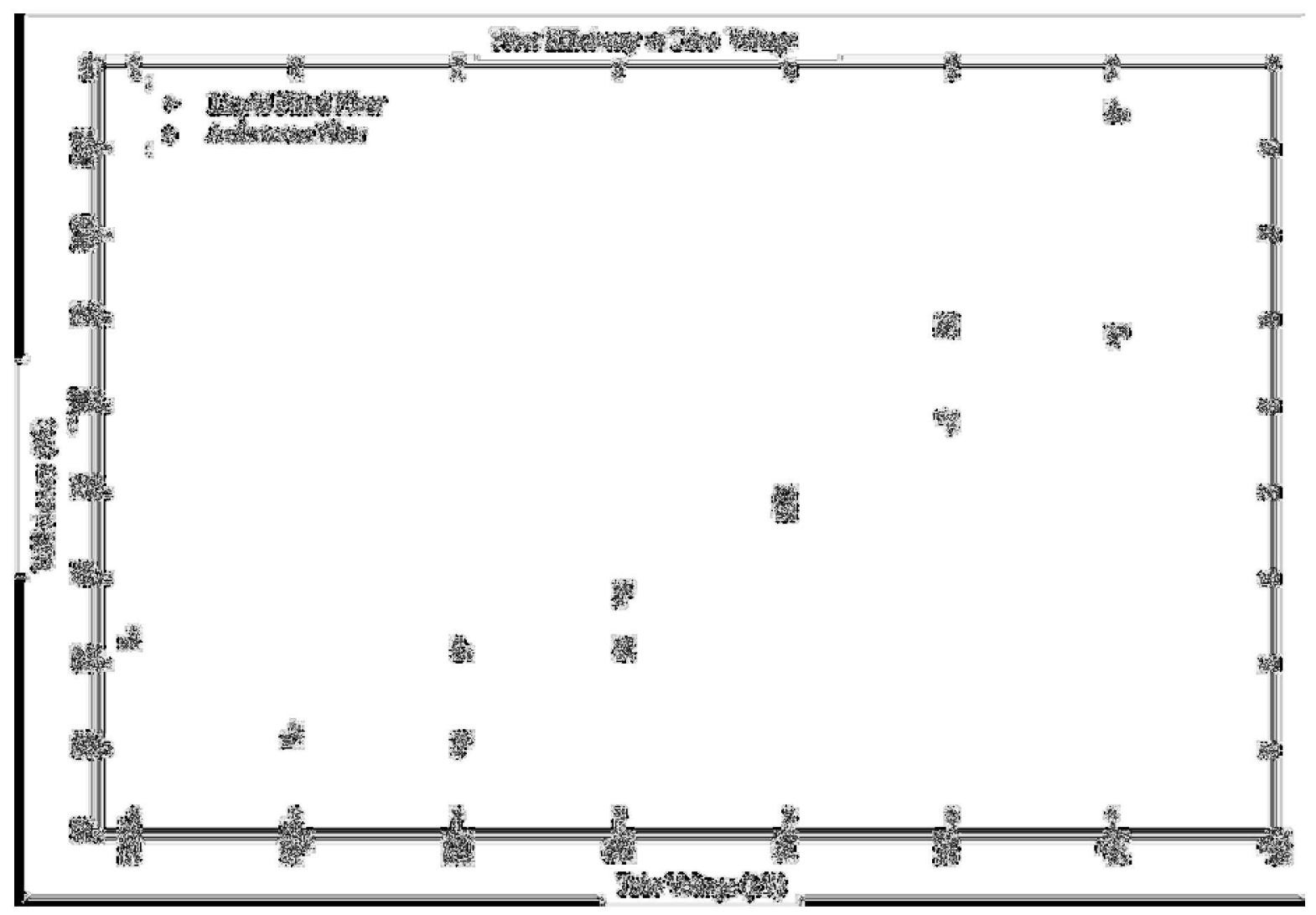

FIGURE 7. Percent efficiency for fiber scintillation photon counts compared to total CdTe photons at discrete tube voltages.

\section{ACKNOWLEDGEMENTS}

The author would like to thank Patty Davis and Warren Kelliher at the NASA Langley Research Center for assistance in this work.

\section{REFERENCES}

1. G. F. Knoll, Radiation Detection and Measurement, publisher, John Wiley \& Sons (2000), Ch 8 \& 14.

2. H. Leutz, "Scintillating Fibers", Nucl. Instr. And Meth., 364, pp 442-448 (1995).

3. A.W Snyder and J.D. Love, Optical Waveguide Theory, publisher, Chapman and Hall (1983), Ch 1,2,13 \& 14.

4. NIST Website: Tables of X-Ray Mass Attenuation Coefficients and Mass-Energy Absorption Coefficients, http://physics.nist.gov/PhysRefData/XrayMassCoef/cover.html.

5. L. Shen and J.A. Kong, Applied Electromagnetism, publisher, PWS Engineering (1987), pp. 225-230.

6. B. Podolsky and K.S. Kunz, Fundamentals of Electrodynamics, publisher, Marcel Dekker (1969), pp. 302-313.

7. R.A. Laudise, Single Crystal Growth, publisher, Prentice-Hall Inc. (1970), Ch 5.

8. J.B. Birks, Theory and Practice of Scintillation Counting, publisher, Paragon Press (1964). 